\title{
Physical Therapy, Speech Therapy and Occupational Therapy: past, present and future
}

\author{
Raquel Aparecida Casarotto, Claudia Regina Furquin de Andrade, \\ Selma Lancman, Clarice Tanaka, Fátima Corrêa Oliver
}

$\mathrm{T}^{\mathrm{s}}$ he mission of the Departament of Physical Therapy, Speech Therapy and Occupational Therapy of the University of the São Paulo Faculty of Medicine (FMUSP) includes undergraduate and graduate education, research and culture and extension of services to the community, related to physical therapy, speech therapy and occupational therapy, according to the highest ethical and moral precepts, with excellence, international relevance and inclusion in the Brazilian reality.

It is similar and compatible with the mission of the USP Faculty of Medicine in all of its aspects. As the department is atypical in its essence, multidisciplinary (three undergraduate courses) the mission of each of the areas of knowledge that form this department is also linked to the departmental mission. The historical aspects and prospects of each course will be presented below, individually.

\section{PHYSICAL THERAPY}

Physiotherapy Practice in Brazil began in 1919, when the Department of Medical Electricity was founded by Professor Raphael de Barros from the University of São Paulo's Faculty of Medicine.

In 1929, doctor Waldo Rolim de Moraes installed the Physical therapy service of the "Arnaldo Vieira de
Carvalho" Radium Institute, located in the facilities of the Central Hospital of the Santa Casa de Misericórdia (Holy House of Mercy). It was also he who planned and installed, in the Clinical Hospital of the University of São Paulo's Faculty of Medicine, the Physical Therapy Service of the São Paulo Clinical Hospital in 1951, who planned the first Physical Therapy Course in Brazil, sponsored by the Raphael de Barros study center, whose purpose was to train technicians in physical therapy. Its facilities were on the 7th floor of the Clinical Hospital's Central Institute and its classes were taught by the hospital's faculty and physicians.

In 1958, the State Law No. 5029 created the National Rehabilitation Institute (Instituto Nacional de Reabilitação - INAR) linked to the Chair of Orthopedics and Traumatology, in partnership with the World Health Organization (WHO), the Pan American Health Organization (PAHO) and the World Confederation for Physical Therapy (WCPT). Thus, the Institute was able to host the first Physical Therapy Course with minimum international standard and duration of two years. Soon after, still in 1958, the INAR became the Rehabilitation Institute (Instituto de Reabilitação - IR), remaining linked to the Chair of Orthopedics and Traumatology (FMUSP). The IR had two functions: the first one was of assistance and focused on the care for people with physical disabilities through integral rehabilitation programs, and the second

Department of Physical Therapy, Speech Therapy and Occupational Therapy, Faculty of Medicine, University of São Paulo. Mailing address: Rua Cipotânea, 51. Cidade Universitária. ZIP CODE: 05360-160. São Paulo, SP, Brasil. email: fofito@usp.br 
one was directly linked to education, with the organization of regular courses for training professionals in the different fields of rehabilitation. In this way the technical courses for Physical Therapy, Occupational Therapy, Orthosis and Prosthetics and Locomotion Assistance for the Blind were created.

The Decree-Law 938 of October 13, 1969 (the date which originated the Physical therapist's Day) was an important milestone for Physical Therapy. Article 2 states that physical therapists who graduated from renowned schools and courses are professionals with higher education and Article 3 defines as the physical therapist's private activity the performance of physical therapy methods and techniques aiming at restoring, developing and preserving the patient's physical capacity. The professional training of the undergraduates of that time focused on the healing and rehabilitation aspects and did not include health promotion and prevention of diseases, and mainly focused on diseases that affected the musculoskeletal and neurological system.

The regulation of the Federal Council of Education, expressed in Report 388/63, acknowledged the Physical therapy courses and determined that they should be three years long, which lasted until 1979. In the following year it started to be four years long.

The USP Physical Therapy Course followed the changes in the workload and in the great expansion of the physical therapist's field of activities, however, the Minimum Physical Therapy Curriculum was only approved on February 28, 1983 through Report No. 622/82 of the Federal Council of Education. The new Minimum Curriculum for the Physical Therapy courses, with 3240 hours to be fulfilled in a minimum period of four years and a set of subjects that ensured the training of professionals with a generalist profile, opposed to the vision of rehabilitation imposed by the curriculum that existed then.

Another accomplishment for the profession was the establishment of the National Curriculum Guidelines for the Physical Therapy Undergraduate Course after four years of discussion, through the Resolution of the CNE/ CES 4 of February 19, 2002. These guidelines define the principles, foundations, conditions and procedures for training physical therapists, established by the Chamber of Higher Education of the National Council of Education for implementation at the national level in the organization, development and evaluation of the educational projects of the Physical Therapy Undergraduate Courses of the Higher Education System's Institutions. The USP Physical Therapy Undergraduate Course followed the recommendations of the Guidelines having as the profile of former undergraduates/professionals the generalist, humanist, critical and reflective Physical therapist, able to work in all the health care levels, based on scientific and intellectual rigor.

In 2005, the Faculty of Medicine acknowledged the academic maturity of the Physical Therapy Course professors and created the first position of Full Professor of the Physical Therapy Course, which was occupied by Prof. Clarice Tanaka.

From 2012 on, the Physical Therapy Course became 5 years long with a workload of 5085 hours.

The USP Physical Therapy Course is currently working in the areas of physical therapy knowledge, focused on health promotion, disease prevention, treatment and rehabilitation: Orthopedics and Traumatology, Rheumatology, Adult's and Children's Neurology, Intensive Care, Cardiology, Pulmonology, Geriatrics, Angiology, Occupational Health, Public Health, Women's Health, Dermatology, Sports Medicine. The acquisition of new professors is in the Course's Target Plan, which aims to expand the following areas: Neonatology and Oncology.

The course is undergoing a process of internationalization taking into account both the exchange of undergraduate students with different universities in the world and the fact that it is welcoming students from other countries, especially from Chile, Colombia and Argentina.

From the research point of view, the international exchange process also happens among Universities from France, Chile, Canada, USA, Australia and Argentina.

Regarding Post-graduation, the course pleads the increase of its score from 5 to 6 in the near future. We also started an inter-institutional collaboration with the Federal University of João Pessoa - PB through the DINTER (Doutorado Interinstitucional - Inter-institutional Doctorate) program of CAPES.

The prospects of the Course for the Future are based on the development of new areas of research in Neonatology, Oncology, Dermatology and Functional and Sports and in advancing in the ongoing internationalization 
process of the course, for the undergraduate course, research and post-graduate courses.

\section{SPEECH THERAPY}

The creation of the Speech Therapy Course in the University of São Paulo's Faculty of Medicine dates back to 1958 . In the period between 1958 and 1975 this course was linked to the FMUSP's Chair of Otolaryngology and Ophthalmology. In a Session on 10/25/1975 the University Council approved and authorized the operation of the Speech Therapy Undergraduate Course, with a university curriculum, and the course was linked to the Department of Clinical Medicine. In the period between 1976 and 1999 there was the acknowledgment of the profession and the creation of the Regional and Federal Councils (12/09/1981, Law 6965). During this period the FMUSP Speech Therapy Course was acknowledged by the Ministry of Education and the minimum content and length of time were set (Resolution 6, 1983).

On $12 / 17 / 1999$, due to the structuring of critical mass and development of the area as a science of human communication, the FMUSP Speech Therapy Course, along with the Physical Therapy and Occupational Therapy Courses, began constituting a Department of Physical Therapy, Speech Therapy and Occupational Therapy (Departamento de Fisioterapia, Fonoaudiologia e Terapia Ocupacional- FOFITO) of the University of São Paulo's Faculty of Medicine.

The period between 1999 and 2015 - where there was the possibility for the Speech Therapy Course and the Speech Therapy Subject (which started in 2001 with the first full professor for the area) to develop - was crucial to the vast expansion of care activities, to academic maturity and autonomy in research management.

Nowadays, the mission of the FMUSP Speech Therapy Course, meeting the guidelines of our university and international standards, is to train individuals in the undergraduate and lato and stricto sensu post-graduate level for professional life. The speech therapist who graduated from USP should be an ethical, competent, reflective professional, who works with expertise and scientifically. He/she is a professional whose work is based on evidence and who respects diversity. The mission of the undergraduate Speech Therapy Course is to train a general Speech therapist who is able to act competently and critically at the several levels of health care. His or her basic general training should be consistent and thorough, building the foundations for the construction of professional knowledge, enabling his or her inclusion in the labor market. This professional will also have the awareness and competence for permanent updating. Besides, students should act in harmony with the tradition in scientific research and their practical training aims at the capacitation and rehabilitation of people - from birth to death - who have any impairment in human communication (hearing, language, neurovegetative system and features of the face).

With the Dean of Undergraduate Studies' approval, we begin in this year of 2016 the first Speech Therapy Undergraduate Course in Brazil, which will grant its first degrees within 5 years. The goal of the extension of the course is that our students' last year can be completely practical with the characteristics of medical internship.

Our mission in lato sensu post-graduation is aimed at continuing education through refresher, specialization and improvement courses, which have started in the 90s already. The stricto sensu post-graduation, due to its academic nature, has been allowing us to train masters and doctors in Rehabilitation Sciences for over 10 years. Since 1996 the professors and students of the FMUSP Speech Therapy Course have been actively involved, along with funding agencies internal and external to the university, in generating research and funded scholarships. Our thinking is towards the pursuit of the development of specific research projects, as well as those which are multi and interdisciplinary and aim at generating knowledge and transferring technology of relevant application at a regional, national and international level (we currently have postdoctorate fellows in several foreign countries, and official agreements were signed with different countries).

The sub-areas of teaching and research in speech therapy are: Audiological Amplification and Rehabilitation for Adults, Audiological Assessment in Adults, Balance Disorders and Vestibular Rehabilitation, Cochlear Implants, Educational Audiology, Electrophysiology, Hearing Conservation and Environmental Audiology, Hearing Disorders, Pediatric Audiology - Identification and Early Intervention, Neurogenic Aspects in Children, 
Adults and the Elderly, Oral Language in Children, Adults and the Elderly, Psychosocial and Psychiatric Aspects in Children, Adults and the Elderly, Articulation and Phonology, Augmentative and Alternative Communication, Aural Rehabilitation, Multilingualism Development and Disorders, Fluency and Fluency Disorders, Literacy, Written Language and Learning, Swallowing and Dysphagia, Voice, Voice Disorders and Diseases, Professional Matters and Management of the Speech Therapy Practice.

The projections and expectations for the future of speech therapy (discussion for the elaboration of the new curriculum of the FMUSP Speech Therapy Course and bases of the initial proposition of the American SpeechLanguage and Hearing Association (ASHA) for 2025 state that the didactic/academic training programs and the clinical training practices should be aligned in order to maximize learning. The goal would be professional training in the ideal basic level (generaliist) with incentives and support to continue learning in post-graduation.

The programs of the "future" should be supported by sensitive goals such as: 1 . Consistency - the educational priorities must be clear and fully disclosed; 2 . Continuity - hierarchical conception between graduation and lato and stricto sensu post-graduation; 3. Common learning homogeneity between students with different backgrounds; 4. Skill-based - emphasis on documented knowledge and on the skills developed and acquired and not in the "hours" added; 5. Community Conscience - construction of ethics in the involvement with community.

To reinforce these goals, the programs should consider: The development of integrated contents; The basic Sciences (anatomy and physiology, linguistics, psychology, etc.); The Multicultural issues; The Use of technology and evidence-based practices; Critical thinking, problem solving and the ability to consider several perspectives; The Personal qualities such as the ability to work as part of a team, to collaborate, to negotiate and to solve conflicts; The Case studies, observation and learning approaches based on issues that provide practical, experiential and long lasting learning; The development of a "common" basis as accumulated evidence to support practices; Clinical Education, which provides "learning by doing it with guidance"; Using the study critically; Expanding the use of distance learning; Creating different levels of certification for the practice; Exposing students to early clinical experience; Being more intentional about teaching dispositions and attitudes through the infusion of such teaching within academic and clinical program elements; Increasing the opportunity for cycle learning; Incorporating preceptor models with a greater focus on mentoring and modeling; Increasing the diversity of students and professors; Preparing and supporting clinical professors, Incorporating practices that support the academic results based on evidence.

A Speech Therapy program for the future should provide and/or increase the knowledge and skills with the following areas: Cognitive sciences, Gerontology, Pharmacology, Language literacy connection, Genetics, Brain imaging, Systems engineering and policy making, Bilingualism, Communication effectiveness, Business practices, Risk management, Regulations/refund issues, Advocacy, Learning throughout life.

Professional preparation for the future involves: A greater length of time (for example, obtaining basic level skills plus an introduction to the specialties selected by the students); Wider, with a more interdisciplinary approach; Different clinical populations; Incorporating new technologies and procedures that require new therapeutic approaches; Enhancing the chances of a clinical doctor to enter a doctorate; More flexibility and dynamism to meet both the needs of the student and the professional development; Greater access to technology, especially regarding simulations/simulators; Less traditional theoretical educational content; Teaching of the financial bases for the provision of services (value of the services, cost of service provision) including regulations/ reimbursement issues; Including more flexible and dynamic recruitment processes to increase internationalization; Promoting cooperative activity among students, teachers and professionals.

\section{OCCUPATIONAL THERAPY}

The FMUSP Occupational Therapy Undergraduate Course is the oldest in the country, the first to be recognized by the World Federation of Occupational Therapy and also the first to feature a faculty completely formed by doctors.

It was structured with the creation in 1956 of the 
National Rehabilitation Institute (Instituto Nacional de Reabilitação - INAR) at the Clinical Hospital which, with incentives from the World Health Organization (WHO), the International Labour Organization (ILO) and the United Nations Organization for Education, Science and Culture (UNESCO) implemented "Centers for Demonstrations of Rehabilitation Techniques". The INAR was composed of Brazilian professionals who were qualified for training occupational therapists through programs in the United States, and later welcomed foreign professionals to develop the manufacture of prosthetics and orthosis, professional rehabilitation and to expand professional training.

In 1958, the INAR became the Rehabilitation Institute (Instituto de Reabilitação - RI) linked to the FMUSP's chair of Orthopedics and Traumatology, where the training of occupational therapists was developed over a period of two years. In 1963, the course started to have a three-year duration and complied with the standards for university education. With the deactivation of the IR, the course was linked to the FMUSP Department of Orthopedics and Traumatology and started operating in the facilities of the Faculty. In 1974 it was incorporated into the Department of Clinical Medicine, its curricular structure was adapted to the academic standards and to graduate training with the incorporation of new fields of activity of the occupational therapist, which were developing in the country.

Only in the 80 s the course, already with a 4-year duration, had access to an infrastructure and faculty compatible with the complexity that the professional training suggested. In 1983, the approval by the Ministry Education and Culture of the minimum curriculum, demanding a consistent specific training center, made it possible to increase, quantitatively and qualitatively, the faculty, which enabled a new inclusion of the area in USP.

Occupational Therapy contributes to the scientific dissemination of the area since 1991, when the issuing of the USP Occupational Therapy Journal, the first journal to be indexed in an international database in Latin America, began. Also in 1991, teaching, research and community service extension activities were propelled with the opening of its own facilities, the Physical Therapy, Speech Therapy and Occupational Therapy Teaching and Research Center on the Butantã campus, which was reaffirmed by the creation, in 1999, of the Department of Physical Therapy, Speech Therapy and Occupational Therapy.

Since the establishment of the undergraduate course, curricular reforms were implemented, which are, to this day, the basis for the creation of other undergraduate courses in the country. It is worth mentioning the training aimed at assistance work in facilities which are external to the hospital, with reflective and innovative educational programs and the development of therapeutic resources and care technologies technically and methodologically contextualized in debates throughout the country. In association with students resources and technologies are improved in order to support the social participation of people and groups that benefit from Occupational Therapy. Over time, these educational perspectives, combined with research and extension, better qualified care in traditional areas of the profession such as mental health, labor and rehabilitation and established other possibilities for their development in the education, culture and social assistance scopes.

A milestone of the expansion of the Area in the University was the first tender for Full Professor in Occupational Therapy, performed in 2009 at the Faculty of Medicine.

The mission of the FMUSP Occupational Therapy is to train qualified professionals for meeting the needs of people presenting (temporary or permanent) restrictions in everyday life, in autonomy, and in participation in social life, due to specific (physical, sensory, intellectual, mental and/or social) issues . Professors and professionals work in the undergraduate and post-graduate courses to train ethical professionals who are technically and scientifically competent, able to make a critical analysis of the historical and social context and to have respect for the cultural, ethnic and gender diversity. These are essential values for the formation, the affirmation of human rights, the interdisciplinarity, interprofessionalism and intersectoriality in public health, social assistance, education, work and culture policies.

The contributions of FMUSP's Occupational Therapy within the scope of the public health services predate the creation of the SUS (Sistema Único de Saúde, the Brazilian National Health System) and are structured so as to qualify care in services of different 
levels of complexity and technological density such as those of hospital, ambulatory and primary care nature. The professionals trained are challenged to participate in programs in the areas of mental health, worker's health, elderly's, children's and adolescent's health, care to disabled people, as well as specialties such as traumatology and orthopedics, neurology, leprosy, among others.

The area has a tradition in the field of service extension with consolidated partnerships in the fields of health, culture, labor, social care and education. It develops actions in hospital contexts (CH- FMUSP and University Hospital); ambulatory contexts and primary health care contexts, and also in community and territorial contexts.

In 2010, the area was part of the national movement for the acknowledgment of Occupational Therapy by the Ministry of Social Development and Fight against Hunger, as one of the 12 professions to include services within the scope of the National Social Assistance Policy and act in the basic social protection, special social protection of medium and high complexity services, which requires the continuous training of professionals.

The FMUSP Occupational Therapy also innovated teaching, research and the extension of services to the community in the area in the country, with the creation of Laboratories of Research on Mental Health, Disability, Rehabilitation, Social Field, Elderly Health, Assistive Technology, Care in Hospital Contexts, as well as on the interfaces between Health and Labor and between Art and Body. These initiatives resulted in specialization courses: Rehabilitation, Artistic Praxis and Social Occupational Therapy, as well as in Programs of Multiprofessional Residency in Health Promotion and Care in Hospital Attention and in Mental Health with emphasis on Drug Addiction.

The research in the area was structured with the participation of professors, since the creation of the PostGraduation Program in Rehabilitation Sciences of the Department at the FMUSP in 2005, and in other programs

\section{REFERENCES}

1. Trigo de Souza LM. Fofito - 50 anos de pioneirismo e lutas. São Paulo: Fundação Faculdade de Medicina; 2008.

2. Departamento de Fisioterapia, Fonoaudiologia e Terapia Ocupacional. Avaliação institucional 2010-2014. São Paulo; 2014. of USP such as Aesthetics and Art History/MAC-USP; USP's Interdisciplinary Training in Health, of School Psychology and Development of the Institute of Psychology and Humanities, Rights and other Legitimacies of the FFLCH/USP, as well as UFSCar's Occupational Therapy and UNESP's Psychology, at the Assis Campus. These alternatives consolidate the teaching and research and ,in the near future, they will implement the proposal for a Professional Masters Course in Occupational Therapy and Social Inclusion Processes, under analysis at the university.

The area has been called to include consultancies in Higher Education, Mental Health and Rehabilitation Assessments, with several ministries and departments. It also built and participates in the National Network of Education and Research in Occupational Therapy, in representations with the Brazilian Association of Occupational Therapists and the Federal and State Occupational Therapy Councils system and integrates boards of directors of scientific societies in related areas. It also establishes dialogues for education and research in France, Portugal, Spain, England, Canada, South Africa, Chile, Colombia and Argentina, where its contributions also reach acknowledgment and prestige.

These are prospects for the future for the Area in FMUSP to maintain the excellence of its ongoing activities and to expand the education and research areas aimed at: Actions in the Cultural Field; Attention to Alcohol and Drugs Users; Hospital Contexts of High Complexity; Pain and Palliative Care; Aging; Ergonomics; School inclusion; Childhood and Adolescence and Specific Technological Resources. The aim is also to increase the number of enrollments in the only public undergraduate course in the city of São Paulo.

The history of Occupational Therapy at FMUSP and the new education and research propositions will further strengthen its contributions to the professional field in the country.

3. Curso de Fisioterapia da FMUSP. Projeto Político Pedagógico (2008-2012). São Paulo; 2012.

4. Curso de Fonoaudiologia da FMUSP. Projeto Político Pedagógico (2013-2016). São Paulo; 2011.

5. Curso de Terapia Ocupacional da FMUSP. Projeto Político Pedagógico (2007-2011). São Paulo; 2011. 